\title{
Perspectives on Improved Therapy of Septicemia by Rapid Bacterial Identification Using MALDI-TOF MS and Rapid Susceptibility Testing
}

\section{Jakovljev $\mathrm{A}^{1,2 *}$ and Bergh $\mathrm{K}^{1,2}$}

${ }^{1}$ Department of Medical Microbiology, St. Olavs Hospital, Trondheim University Hospital, Trondheim, Norway

${ }^{2}$ Department of Laboratory Medicine, Children's and Women's Health, Faculty of Medicine, Norwegian University of Science and Technology, Trondheim, Norway

\section{Commentary}

The most serious clinical manifestations of bloodstream infections (BSI), sepsis and septic shock, are urgent conditions requiring prompt initiation of adequate antimicrobial therapy [1]. In septic shock, every hour of delayed upstart of effective antimicrobial therapy was associated with a decrease in survival rate, thus falling from $82.7 \%$ initially to $42 \%$ after six hours of hypotension duration [2]. Rapid identification of microorganisms directly from blood cultures after flagging positive is the first step in shortening the diagnostic process. A number of molecular methods for direct identification from blood cultures have been described, including multiplex real-time PCR [3]. After matrixassisted laser desorption ionization- time of flight (MALDI-TOF) mass spectrometry (MS) was introduced in microbiological laboratories in the last decade [4], development of rapid in-house [5,6] and commercial methods (Sepsityper Kit, Bruker Daltonics, Germany) for direct identification of organisms from positive blood cultures have been reported.

We have previously described the development of two in-house methods for rapid identification of microorganisms directly from positive blood cultures by MALDI-TOF MS [7]. A modification of the method described by Martini et al. [8] by adding a formic acid extraction step directly on the MALDI-TOF plate provided the higher level of identification obtained by the first in-house method. By adjustment of cut-off score values to $>1.7$ for species and $>1.5$ for genus level identification, $89.3 \%$ of organisms were identified, including $94 \%$ of Gram negative and $85.4 \%$ of Gram positive bacteria. Overall concordance to definitive identification was $99.3 \%$ to genus and $96.6 \%$ to species level. Genus discrepancy was observed only in one case of Citrobacter freundii misidentified as Lactobacillus helveticus, with the score 1.513 close to the lower limit. Five minor discrepancies were observed in differentiating among Streptococcus mitis group isolates and insufficient identification of Salmonella to species level, which are known limitation of MALDI-TOF MS identifications.

Due to its excellent applicability and reliability, this rapid in-house method providing identification results in less than 30 minutes, is now implemented in our routine laboratory work. Since September 2015, the laboratory opening hours are extended to $10 \mathrm{pm}$ and positive blood cultures are analyzed directly by MALDI-TOF MS three times a day. After updating of Bruker Daltonics database in October of 2015, all analysis are being performed on the new software Compass 4.1 comprising 5989 different microorganisms strains in its database (5291 bacterial strains and 691 eukaryotic-mainly fungal-strains). A higher level of confident identification to species level with score values $>2.0$ was noticed since updating, including less frequent organisms as Pasteurella multocida, Bacillus cereus group, Burkholderia cepacia complex. Almost all isolates of Enterobacteriaceae, Pseudomonas spp, staphylococci, $\beta$-hemolytic streptococci, enterococci and anaerobes are now routinely reliably identified directly with confident genus and species score values, $>1.7$ and $>2.0$, respectively. Identification problems are still noticed with Propionibacterium spp. and Corynebacterium spp., supposed to be caused by insufficient concentration of organisms in blood culture flasks. In our routine laboratory work, this observation have led to exclusion from direct identification of those almost always single positive blood culture flasks flagging positive after 5 days of incubation as probable contaminants. Direct identification of Pneumococci is still based on Gram microscopy and agglutination test due to previously described problems [7,9].

The most important purpose of rapid direct identification in our laboratory was gaining information leading to adequate antimicrobial therapy in septic patients, based on knowledge of intrinsic antimicrobial resistance and national resistance patterns of the bacterial species identified [10]. In our laboratory, the results of antimicrobial susceptibility testing (AST) are still obtained the next day after the blood cultures are flagged positive. The method presently used is direct disk diffusion test from blood culture flasks, where resistance results are read after 18 hours of incubation. Briefly, different volumes of blood from blood culture flasks are added to $3 \mathrm{ml}$ of $0.9 \%$ sterile saline (volume dependent on results of Gram stain and direct MALDI-TOF MS species identification), floated on adequate resistance agar media by swabbing followed by placing of antibiotic disks on the surface. The interpretation of results is performed by using NordicAST breakpoints (version 6.0; 2016) based on European Clinical Antimicrobial Susceptibility Testing Breakpoints (EUCAST), but with the comments adjusted to local Nordic antimicrobial patterns. This method has been employed for several years in our laboratory, validated internally and assessed as reliable in comparison with AST performed from bacterial growth after subcultivation on agar media. Direct disk diffusion method was recently compared with the VITEK-2 based definitive AST and acceptable accuracy of the disk diffusion test system was reported [11].

Due to increasing number of resistant bacterial isolates, especially those with plasmid transferable resistance genes, more rapid AST results are required. We have considered different options for this approach, and one that could be readily employed in our routine laboratory work is early reading of direct disk diffusion test results, approximately after 6 hours of incubation. This approach is widely used in some laboratories for getting rapid preliminary AST results, but publications based on validation of this method are scarce.

*Corresponding author: Aleksandra Jakovljev, MD, Microbiologist, Departmen of Medical Microbiology, St. Olavs Hospital, Trondheim University Hospital Trondheim, Norway; Kăre Bergh, Professor, PhD, MD, Microbiologist, Department of Laboratory Medicine, Children's and Women's Health, Faculty of Medicine Norwegian University of Science and Technology, Trondheim, Norway, E-mail: aleksandra.jakovljev@stolav.no; kare.bergh@ntnu.no

Received April 27, 2016; Accepted May 05, 2016; Published May 15, 2016

Citation: Jakovljev A, Bergh K (2016) Perspectives on Improved Therapy of Septicemia by Rapid Bacterial Identification Using MALDI-TOF MS and Rapid Susceptibility Testing . J Antimicro 2: 115. doi:10.4172/2472-1212.1000115

Copyright: @ 2016 Jakovljev A, et al. This is an open-access article distributed under the terms of the Creative Commons Attribution License, which permits unrestricted use, distribution, and reproduction in any medium, provided the original author and source are credited. 
Recently, oral and poster presentations [12] from Kahlmeter et al. at ESCMID 2016 reported the results of disk diffusion AST directly from blood cultures read at different time points: after 6,8 and 12 hours of incubation. The conclusion was that regular breakpoints cannot be applied if early AST reading is performed as the corridor between wild type and non-wild type population widens with longer incubation time, and some resistance mechanisms can be expressed only after the recommended length of incubation. These researchers are currently working on establishment of breakpoints for early disk diffusion AST reliable reading. Automated AST options (Phoenix system, Vitek 2) were reported as successful for direct AST of Gram negative bacterial isolates from blood cultures $[13,14]$. The results of our internal validation of AST using automated Phoenix system performed on Gram negative bacterial urine isolates during autumn 2015 were more similar to those reported by Romero-Goméz et al. [15], with sporadic very major and major errors for cefuroxime, piperacillin-tazobactam and trimethoprim-sulfamethoxazole.

Considering our requirements for rapid detection of resistant isolates, the major resistance problems are associated to Escherichia coli and Klebsiella pneumoniae isolates bearing plasmid located genes for extended-spectrum $\beta$-lactamase (ESBL) enzyme production, represented mainly by CTX-M enzymes [16]. ESBL producing isolates are resistant to most of $\beta$-lactam antibiotics, except carbapenems and rapid adjustment of empirical antimicrobial therapy is necessary. The prevalence of ESBL producing E. coli and K. pneumoniae isolated from blood cultures in Norway is still low, reported to be $5.8 \%$ and $3.9 \%$, respectively, in 2014 [10]. Due to more travel activity and migration this figure is expected to increase. The few isolates of carbapenemase producing bacteria diagnosed in Norway were imported cases $[17,18]$. Molecular diagnostics of ESBL CTX-M, TEM, SHV and CARBA KPC, OXA-48, VIM , NDM genes for detection of ESBL and carbapenemase producing bacteria by real-time PCR is feasible by commercial tests and in-house methods $[19,20]$, but is considered to be an expensive approach requiring both time, facilities and in many instances trained personell. Additionally, PCR will only detect the known genes encoding most frequent enzymes, while resistance caused by other mechanisms (e.g. porin modification, efflux pump) will not be detected.

Development and evaluation of inexpensive, reliable and rapid methods for direct detection of ESBL and carbapenemase producing bacteria from positive blood cultures by using MALDI-TOF MS [2126] warrants consideration. This approach is based on detection of cephalosporine or carbapenem degradation into hydrolyzed products. Establishment of software database for detection of specific peaks is necessary for this method, which then may provide results within 1 to 4 hours. The results obtained in these studies varied in relation to class of ESBL or carbapenemases detected and whether resistance was chromosomally or plasmid encoded, but were generally assessed promising. In the study performed by Mirande et al., the attempt to understand the kinetics of hydrolysis of antibiotics was done by monitoring the detection of hydrolysis of ertapenem and faropenem by using the VITEK MS RUO system during 24 hours. The authors presented an interesting discussion about strengths and weaknesses of previously described MALDI-TOF MS protocols with respect to applicability and reliability for work in routine microbiological laboratories [27].

Nordmann et al. [28] recently described a test for rapid diagnostic of ESBL-producing Enterobacteriaceae based on biochemical detection of hydrolysis of the $\beta$-lactam ring of cefotaxime. This test, named
Nordmann-Dortet-Poirel (NDP) test was recently evaluated by Dortet et al. [29] for rapid detection of ESBL-producing Enterobacteriaceae directly from blood cultures. The results of NDP testing compared with molecular diagnostic techniques have shown complete concordance and ESBL NDP test had excellent sensitivity and specificity of $100 \%$. This inexpensive test is suitable for bench work and can be directly performed on positive blood cultures with results provided within 30 minutes. These encouraging results should be confirmed in other laboratories. The rapid test (CARBA-NP Assay) based on the similar biochemical principle was developed by the same group of authors for rapid detection of carbapenemase producing Enterobacteriaceae and evaluated also directly from blood cultures with sensitivity and specificity of $97.9 \%$ and $100 \%$ respectively [30]. However, these excellent results were not completely reproduced in the study performed by Chong et al. [31] where MALDI-TOF MS detection of carbapenemase activity in Enterobacteriaceae, Pseudomonas aeruginosa and Acinetobacter spp. was compared against CARBA-NP-Assay. MALDI-TOF MS based diagnostic have shown better performance by detecting carbapenemase activity from $99 \%$ of tested isolates, while CARBA-NP assay detected activity from $85 \%$ of isolates. From the aspect of clinical microbiologists, both MALDI-TOF MS based diagnostics and CARBA-NP-Assay methods are considered attractive due to its rapidness, cost effectiveness and reliability.

The increase of antimicrobial resistance worldwide is worrying, and to improve patient care clinical microbiologists should emphasise the impact of rapid methods for identification and AST of bacteria causing BSI. The field of rapid methods for AST is gaining wide interest, and an array of future technologies, including microarray, nanotechnology and whole genome sequencing, are expected to contribute significantly [32]. In this report we have focused on the combined use of direct MALDI-TOF MS identification from positive blood cultures and rapid cost effective AST methods that can be readily implemented in routine laboratory work providing reliable results.

\section{References}

1. Seifert $H$ (2009) The Clinical Importance of Microbiological Findings in the Diagnosis and Management of Bloodstream Infections. Clin Infect Dis 48: 238 245.

2. Kumar A, Roberts D, Wood KE, Light B, Parrillo JE, et al. (2006) Duration of hypotension before initiation of effective antimicrobial therapy is the critical determinant of survival in human septic shock. Crit Care Med 34: 1589-1596.

3. Lehmann LE, Hunfeld KP, Emrich T, Haberhausen G, Wissing H, et al. (2008) A multiplex real-time PCR assay for rapid detection and diferentiation of 25 bacterial and fungal pathogens from whole blood samples. Med Microbiol Immunol 197: 313-324

4. Seng P, Drancourt M, Gouriet F, La Scola B, Fournier PE, et al. (2009) Ongoing Revolution in Bacteriology: Routine Identification of Bacteria by Matrix-Assisted Laser Desorption Ionization Time-of-Flight Mass Spectrometry. Clin Infect Dis 49: 543-551.

5. Drancourt M (2010) Detection of microorganisms in blood specimens by using matrix assisted laser desorption ionization time-of-flight mass spectrometry: a review. Clin Microbiol Inf 16: 1620-1625.

6. Stevenson LG, Drake SK, Murray PR (2010) Rapid identification of bacteria in positive blood culture broths by matrix-assisted laser desorption ionization-time of flight mass spectrometry. J Clin Microbiol 48: 444-447.

7. Jakovljev A, Bergh K (2015) Development of a rapid and simplified protocol for direct bacterial identification from positive blood cultures by using matrix assisted laser desorption ionization time-of- flight mass spectrometry. BMC Microbiol 15: 258.

8. Martiny D, Dediste A, Vandenberg O (2012) Comparison of an in-house method and the commercial Sepsityper kit for bacterial identification directly 
Citation: Jakovljev A, Bergh K (2016) Perspectives on Improved Therapy of Septicemia by Rapid Bacterial Identification Using MALDI-TOF MS and Rapid Susceptibility Testing . J Antimicro 2: 115. doi:10.4172/2472-1212.1000115

Page 3 of 3

from positive blood culture broths by matrix-assisted laser desorption-ionisation time-of-flight mass spectrometry. Eur J Clin Micriobiol Infect Dis 31: 2269-2281.

9. Prod'hom G, Bizzini A, Durussel C, Bille J, Greub G (2010) Matrix-assisted laser desorption ionization -time of flight mass spectrometry for direct bacterial identification from positive blood culture pellets. J Clin Microbiol 48: 1481-1483.

10. NORM/NORM-VET (2014) Usage of Antimicrobial Agents and Occurrence of Antimicrobial Resistance in Norway. Veterinaeinstitute.

11. Stokkou S, Geginat G, Schlüter D, Tammer I (2015) Direct disk diffusion test using European Clinical Antimicrobial Suseptibility Testing Breakpoints provides reliable results compared with the standard method. Eur J Microbiol Immunol 5: 103-111.

12. ESCMID eLibrary (2016) Tentative breakpoints for early reading of disk diffusion tests for Escherichia coli, Klebsiella pneumoniae, Staphylococcus aureus and Streptococcus pneumoniae.

13. Funke G, Funke-Kissling P (2004) Use of the BD PHOENIX Automated Microbiology System for Direct Identification and Susceptibility Testing of Gram-Negative Rods from Positive Blood Cultures in a Three-Phase Trial. J Clin Microbiol 42: 1466-1470.

14. Wimmer JL, Long SW, Cernoch P, Land GA, Davis JR, et al. (2012) Strategy for Rapid Identification and Antibiotic Susceptibility Testing of Gram-Negative Bacteria Directly Recovered from Positive Blood Cultures Using the Bruker MALDI Biotyper and the BD Phoenix System. J Clin Microbiol 50: 2452-2454.

15. Romero-Gómez MP, Rosa Gómez-Gil R, Pano-Pardo JR, Mingorance J (2012) Identification and susceptibility testing of microorganism by direct inoculation from positive blood culture bottles by combining MALDI-TOF and Vitek-2 Compact is rapid and effective. J Infect 65: 513-520.

16. Cantón R, Coque TM (2006) The CTX-M b-lactamase pandemic. Curr Opin Microbiol 9: 466-475

17. Glasner C, Albiger B, Buist G, Tambić Andrašević A, Cantón R, et al. (2013) Carbapenemase-Producing Enterobacteriaceae In Europe: A Survey Among National Experts From 39 Countries, February 2013. Euro Surveill 18.

18. Cantón R, Akóva M, Carmeli Y, Giske CG, Glupczynski Y, et al. (2012) Rapid evolution and spread of carbapenemases among Enterobacteriaceae in Europe. Clin Microbiol Infect 18: 413-431.

19. Poirel L, Walsh TR, Cuvillier V, Nordmann P (2011) Multiplex PCR for detection of acquired carbapenemase genes. Diag Microbiol Infect Dis 70: 119-123.

20. Monteiro J, Widen RH, Pignatari ACC, Kubasek C, Silbert S (2012) Rapid detection of carbapenemase genes by multiplex real-time PCR. J Antimicrob Chemother 67: 906-909.
21. Carvalhaes CG, Cayo R, Visconde MF, Barone T, Frigatto EAM, et al. (2014) Detection of carbapenemase activity directly from blood culture vials using MALDI-TOF MS: a quick answer for the right decision. J Antimicrob Chemother 69: $2132-2136$.

22. Jung JS, Popp C, Sparbier K, Lange C, Kostrzewa M, et al. (2014) Evaluation of Matrix-Assisted Laser Desorption lonization-Time of Flight Mass Spectrometry for Rapid Detection of -Lactam Resistance in Enterobacteriaceae Derived from Blood Cultures. J Clin Microbiol 52: 924-930.

23. Burckhardt I, Zimmermann S (2011) Using Matrix-Assisted Laser Desorption Ionization-Time of Flight Mass Spectrometry To Detect Carbapenem Resistance within 1 to 2.5 Hours. J Clin Microbiol 49: 3321-3324.

24. Sparbier K, Schubert S, Weller U, Boogen C, Kostrzewa M (2012) MatrixAssisted Laser Desorption Ionization-Time of Flight Mass SpectrometryBased Functional Assay for Rapid Detection of Resistance against $\beta$-Lactam Antibiotics. J Clin Microbiol 50: 927-937.

25. Foschi C, Compri M, Smirnova V, Denicolò A, Nardini P, et al. (2016) Ease-ofuse protocol for the rapid detection of third-generation cephalosporin resistance in Enterobacteriaceae isolated from blood cultures using matrix-assisted laser desorption ionizatione time-of-flight mass spectrometry. J Hosp Infect pp: 140S147.

26. Johansson Å, Nagy E, Sóki J (2014) Instant screening and verification of carbapenemase activity in Bacteroides fragilis in positive blood culture, using matrix-assisted laser desorption ionization-time of flight mass spectrometry. Med Microbiol 63: 1105-1110.

27. Mirande C, Canard I, Buffet Croix Blanche S, Charrier JP, van Belkum A, et al (2015) Rapid detection of carbapenemase activity: benefits and weaknesses of MALDI-TOF MS. Eur J Clin Microbiol Infect Dis 34: 2225-2234.

28. Nordmann P, Dortet L, Poirel L (2012) Rapid Detection of Extended-SpectrumLactamase-Producing Enterobacteriaceae. J Clin Microbiol 50: 3016-3022.

29. Dortet L, Poirel L, Nordmann P (2015) Rapid Detection of ESBL-Producing Enterobacteriaceae in Blood Cultures. Emerg Infect Dis 21: 3.

30. Dortet L, Brechard L, Poirel L, Nordmann P (2014) Rapid detection of carbapenemase-producing Enterobacteriaceae from blood cultures. Clin Microbiol Infect 20: 340-344.

31. Chong PM, McCorrister SJ, Unger MS, Boyd DA, Mulvey MR, et al. (2015) MALDI-TOFMS detection of carbapenemase activity in clinical isolates of Enterobacteriaceae spp., Pseudomonas aeruginosa, and Acinetobacter baumannii compared against the Carba-NP assay. J Microbiol Meth 111: 21-23.

32. Pulido MR, García-Quintanilla M, Martín-Peňa R, Cisneros JM, McConnell MJ (2013) Progress on the development of rapid methods for antimicrobia susceptibility testing. J Antimicrob Chemother 68: 2710-2717. 